\title{
Prospective surveillance study of medium chain acyl-CoA dehydrogenase deficiency in the UK
}

\author{
R J Pollitt, J V Leonard
}

\begin{abstract}
Background-Medium chain acyl-CoA dehydrogenase (MCAD) deficiency is a common disorder of fatty acid oxidation in north west Europe. It is very variable in its clinical consequences and is believed to be considerably underdiagnosed.

Objective-To investigate the diagnosis and outcome of MCAD deficiency in the UK.

Method-A prospective surveillance study through the British Paediatric Surveillance Unit.

Results-Of 62 affected individuals identified, 57 were from England, giving an incidence of 4.5 cases $/ 100000$ births. Forty six cases presented with an acute illness (10 of whom died), 13 cases were identified because of family history, and three for other reasons. Six of the survivors were neurologically impaired.

Conclusions-Despite increased clinical awareness, the mortality and morbidity from MCAD deficiency remain high. The frequency and severity of the disease support the case for the introduction of universal neonatal screening in England and Scotland.

(Arch Dis Child 1998;79:116-119)
\end{abstract}

Keywords: medium chain acyl-CoA dehydrogenase deficiency; screening

Medium chain acyl-CoA dehydrogenase (MCAD) deficiency ${ }^{1}$ is an autosomal recessive disorder of mitochondrial fatty acid oxidation. It is relatively common in some European populations and those derived from them. ${ }^{2}$ In parts of the UK the calculated birth incidence exceeds one in $10000 .^{3}$

The consequences of MCAD deficiency are very variable. Usually, symptoms occur only during periods of catabolic stress, when the impaired fatty acid oxidation pathway is unable to meet demand. This results in hypoketotic hypoglycaemia, often accompanied by hyperammonaemia and increased plasma transaminases. Typically, such attacks follow a febrile illness and comprise lethargy, vomiting, and encephalopathy, leading to seizures, apnoea, cardiac arrest, and death. ${ }^{4}$ Hepatomegaly might be a prominent feature and a diagnosis of Reye syndrome may be considered. Many affected infants die unexpectedly after an apparently minor illness and the cause of death might be classified as sudden infant death syndrome. Overall mortality rates of $\sim 25 \%$ have been reported in two large studies. ${ }^{45}$ However, some MCAD deficient individuals remain asymptomatic, ${ }^{6}$ presumably because they have never been exposed to sufficient catabolic stress to precipitate metabolic decompensation.

Acute episodes in MCAD deficiency are treated by intravenous glucose and if treatment is started early enough the child improves rapidly. Long term management concentrates on ensuring adequate calorific intake and avoidance of fasting, particularly during infectious episodes. ${ }^{1}$ It is believed that crises can be almost entirely prevented by these measures. In the series reported by Iafolla and colleagues ${ }^{4}$ there were no deaths following diagnosis. Thus, there are clear benefits in diagnosing MCAD deficiency presymptomatically.

Mass neonatal screening for MCAD deficiency has frequently been discussed and, with the analysis of acylcarnitines in dried blood spot samples using tandem mass spectrometry, this has now become a practical possibility. ${ }^{78}$ For a screening test to be acceptable, it should fulfil a number of criteria, ${ }^{910}$ and any formal evaluation requires data on incidence, natural history, and the likely effects of earlier diagnosis on outcome. ${ }^{11}$ There have been numerous literature reports of individual cases of MCAD deficiency (reviewed by Touma and Charpentier ${ }^{5}$ ) but the only large series ${ }^{4}$ comprises cases referred to a diagnostic laboratory from a large area and over a long time period. There are considerable difficulties in extrapolating from such data to the current situation in the UK.

Therefore, we have investigated prospectively the diagnosis and outcome of MCAD deficiency in collaboration with the British Paediatric Surveillance Unit. Preliminary data have been presented in a recent assessment of the case for expanding neonatal screening to include a wider range of disorders. ${ }^{11}$

\section{Methods}

MCAD deficiency was included on the Surveillance Unit orange card between March 1994 and March 1996, inclusively (25 months). These cards are sent monthly to all consultant paediatricians in the UK and the Republic of Ireland and are accompanied by short case definitions of the disorders currently under surveillance. The average return rate in 1995 was $94.4 \% .^{12}$ All initial notifications were followed up by a brief questionnaire requesting patient details, presentation, family history, and diagnostic criteria.

The Surveillance Unit returns were supplemented by two circulations to UK laboratories that were likely to have diagnosed MCAD deficiency. These were asked to confirm the 
Table 1 Reasons for investigation for MCAD deficiency

\begin{tabular}{lc}
\hline Indication & $\begin{array}{l}\text { Number } \\
\text { of patients }\end{array}$ \\
\hline $\begin{array}{l}\text { Previous family history } \\
\text { Sibling known to have MCAD } \\
\quad \text { deficiency }\end{array}$ & $6^{\star}$ \\
$\quad$ Sibling suspected to have died of \\
$\quad$ MCAD deficiency & 1 \\
Sibling of newly diagnosed case & 6 \\
Presentation with an acute illness & $36 \dagger$ \\
$\quad$ Survived & $10 \ddagger$ \\
$\quad$ Diagnosed postmortem & 3 \\
Others & 62 \\
Total &
\end{tabular}

${ }^{\star}$ Including one prenatal diagnosis. ${ }^{20}$

tOne with a previously diagnosed sibling but erroneously believed to be unaffected.

$\ddagger$ One neonatal death with previously diagnosed sibling.

Others: One case identified at 2 years of age during investigation for learning difficulties; one reclassified from a diagnosis of glutaric aciduria type 2 made some years previously; and one investigated because of an apparently unrelated congenital abnormality.

diagnoses attributed to them and for details of any missing cases.

\section{Results}

One hundred and three initial notifications were received through the Surveillance Unit. Follow up led to the identification of 55 patients. This total excluded a child of an American family serving in the US air force; a child who was seen in the UK but who had been diagnosed in Germany, where his family was stationed with UK armed forces; one case where the diagnosis was not adequately confirmed; and six cases that had been reported retrospectively (diagnosed before February 1994). Much of the discrepancy between the number of returns and the number of patients was a result of multiple reporting, reflecting the tendency for MCAD deficient patients to be referred to specialist centres either before or after diagnosis. The laboratories reported an additional seven cases, giving 62 in total. Fifty seven of the cases were diagnosed in England and five in Scotland; no reports were received from Wales, Northern Ireland, or the Irish Republic.

Table 1 summarises the reasons for diagnostic investigation. Forty six of the patients were investigated as a result of an acute metabolic decompensation or unexpected death. Hypoglycaemia was recorded in 37 of these. Encephalopathy was noted in 23 cases, although criteria for reporting this may have been interpreted differently because some of the duplicate reports disagreed.

In the 46 cases that presented with acute illness, 39 were diagnosed after a single episode, all but four within 30 days. Six patients were diagnosed after a second acute episode,

Table 2 Outcome of MCAD deficient patients at the time of the study

\begin{tabular}{lc}
\hline Outcome & $\begin{array}{l}\text { Number (\%) } \\
\text { of patients }\end{array}$ \\
\hline Asymptomatic & $15(24 \%)$ \\
Full recovery from attack & $30(48 \%)$ \\
Neurological impairment/developmental delay & $6(10 \%)$ \\
Death & $10(16 \%)$ \\
No information & $1(2 \%)$ \\
Total & $62(100 \%)$ \\
\hline
\end{tabular}

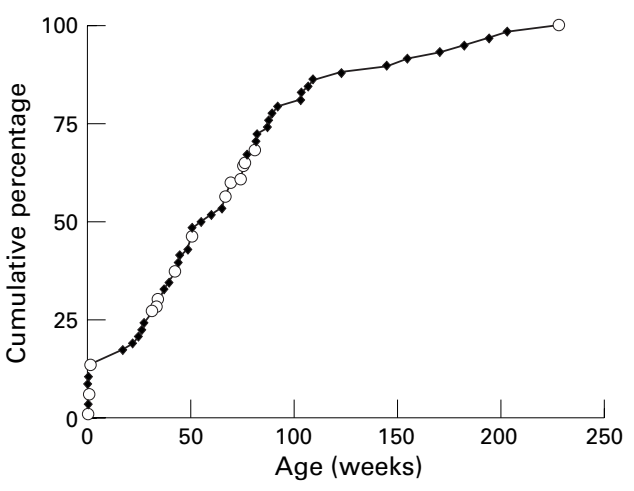

Figure 1 Age of patients at their first episode diagnosed with or suspected of having $M C A D$ deficiency $(n=56)$. Fatal episodes are shown by open circles (some of the data points are obscured by overlap.) Information is missing on two cases diagnosed postmortem.

between 39 and 369 days after the initial illness, and one patient was diagnosed after his third acute episode at the age of 12 years. Table 2 summarises the outcome at the time of reporting.

The 62 cases diagnosed during the period of our study came from 54 families. These families contained another six confirmed cases of MCAD deficiency, diagnosed before this study, and five other children had died in infancy, all of whom probably had MCAD deficiency. Including these, 58 infants had had one or more metabolic crises or had died suddenly; the ages at which the first such event occurred were reported in 56 cases and are plotted in fig 1 . Seven patients, three of whom died, became ill in the neonatal period.

DNA analysis for the common (G985) MCAD mutation ${ }^{2}$ had been performed in 45 families. In 36, the affected children were homozygous for G985, and in the other nine families the children were heterozygous. Thus, $90 \%$ of mutant alleles were G985, in agreement with previous estimates. ${ }^{13}$ The sex ratio in confirmed cases was 1.0.

\section{Discussion}

This study has three main limitations: (1) it is based on diagnosed cases only, estimating minimum incidence but giving no firm data on the proportion of cases that remain undiagnosed or asymptomatic; (2) the short follow up period probably leads to an underestimate of the frequency of long term sequelae; and (3) some data are incomplete and there may be systematic underreporting of symptoms in the newborn period. Nevertheless, the survey has provided sufficient information on the current status of MCAD deficiency in the UK to allow comparison with other reported experience and to confirm the continuing high rate of mortality.

INCIDENCE

There has been much debate about the true incidence of symptomatic cases of MCAD deficiency. An estimate of the incidence of the biochemical condition can be calculated from the G985 heterozygote frequency of the population in question, correcting for the observed frequency of G985 in diagnosed cases. Almost 
universally, the number of cases diagnosed is substantially lower than the calculated incidence. $^{3}{ }^{14-16}$ The shortfall must be accounted for by asymptomatic individuals and/or by a low rate of diagnosis of symptomatic cases, but the relative magnitude of these effects is unknown. Asymptomatic cases appear to be moderately common. The father in one of the families included in this survey was homozygous for the G985 mutation but had never had any symptoms. ${ }^{6}$ In three other families, diagnosis of the index case led to the identification of MCAD deficiency in four siblings over 2 years of age (range, 6-23 years) who had not had significant metabolic decompensation, although in two sisters there had been indications of fasting intolerance. ${ }^{17}$ In four families with an unexplained infant death, MCAD deficiency was diagnosed in a subsequent symptomatic sibling. The only evidence that most untreated cases of MCAD deficiency will eventually have symptoms comes from experience in the Trent region in the UK. Here, MCAD deficiency has been of long standing interest and the number of cases diagnosed in an eight year period was close to the number calculated from heterozygote frequency. ${ }^{3}$

There is a geographical variation in the incidence of MCAD deficiency within the British Isles. The lack of reported cases from the Irish Republic probably reflects the relative rarity of MCAD deficiency which, calculated on the basis of the G985 gene frequency, is approximately 1.7 in 100000 births (PD Mayne, Temple Street Children's Hospital, Dublin, personal communication, November 1997). Our survey reported 4.0 cases/100 000 births in Scotland, despite a fairly low frequency of the G985 mutation in West Scotland. ${ }^{18}$ For England as a whole, 4.5 cases $/ 100000$ births were reported. These figures might overestimate the "steady state" rate of diagnosis because two siblings were diagnosed rather late, 10 years after the first episode in the index case, while another family with three affected siblings was investigated for MCAD deficiency seven years after the death of the index case. ${ }^{17}$ Calculated incidences of MCAD deficiency are 11.3/100 000 for Shropshire, Herefordshire, and Worcestershire and 4.5/100 000 for the Trent region. ${ }^{3}$ Although there is probably still some degree of underdiagnosis in the UK, comparison of the current overall figures with the diagnosis rate of $1.8 / 100000$ in the West Midlands region in 1987-94 (16\% of the expected number) ${ }^{3}$ suggests that a greater proportion of MCAD deficient children than previously are being identified.

CLINICAL PRESENTATION

All but two of the clinically affected cases reported presented with typical symptoms of MCAD deficiency. ${ }^{4}$ The age at first episode ranged from 2 days (three cases) to 4.39 years, with a median of 1.1 years (fig 1 ). Neonatal presentation of MCAD deficiency is being increasingly recognised, ${ }^{19}{ }^{20}$ but the proportion recorded in this survey (seven in a total of 73 suspected or confirmed cases) is much lower than that found in the New South Wales series, where eight of the 20 patients had had significant hypoglycaemia requiring at least intravenous glucose at an age of $1-3$ days. ${ }^{14}$ It is likely that neonatal episodes were significantly underreported in this survey because there was no systematic review of neonatal records.

DIAGNOSIS AND OUTCOME

The only previous study of unselected patients with MCAD deficiency from a defined population is that of Wilcken et al in New South Wales. ${ }^{14}$ The series of Iafolla et al was based on a diagnostic service, although 112 of the 120 patients surveyed were from the USA. ${ }^{4}$ Both these studies were retrospective, spanning up to 17 years and, not surprisingly, current UK experience with diagnosis appears to be better: $35 \%$ of the patients described by Iafolla et al and $25 \%$ of those described by Wilcken et al had more than one episode before diagnosis, compared to $11 \%$ in our study. More than half of the patients in our study who presented with an acute episode were diagnosed within 30 days, compared with an average delay of 1.8 years in the series of Iafolla et al. This probably reflects increased clinical awareness of MCAD deficiency and improved access to appropriate laboratory services. Despite the improvement in diagnosis and paediatric intensive care over recent years, mortality remains high. Ten of the 62 cases diagnosed during our survey period died during the initial attack and if affected siblings and suspicious deaths are included mortality of affected children in these families was 17 of 73 . This compares with mortality rates of $19 \%$ and $25 \%$ in the New South Wales and USA studies, respectively.

Both the New South Wales series ${ }^{14}$ and the USA series ${ }^{4}$ record high levels of disability in patients surviving an acute attack. Five of the 15 New South Wales survivors had some degree of handicap. The USA survivors showed cerebral palsy (9\%), seizure disorder $(14 \%)$, muscle weakness $(16 \%)$, speech disability $(22 \%)$, and/or abnormal results on formal psychodevelopmental assessment $(32 \%)$. In our survey, three of the 36 patients surviving one or more attacks showed signs of neurological damage in the immediate postrecovery period and three others had developmental delay or learning difficulty. These apparently good results may be spurious, however, because a longer follow up period would be necessary to reveal the milder neurodevelopmental deficits.

Our conclusion from this survey is that, despite increasing awareness of MCAD deficiency and more efficient diagnosis, mortality remains essentially unchanged and morbidity is still considerable. Although a few are diagnosed in the neonatal period, most patients have their first detected attack after the age of 3 months and would benefit from early diagnosis by neonatal screening. The number of cases reported suggests that the frequency of MCAD deficiency in England and Scotland is high enough to justify inclusion in the neonatal screening programme. ${ }^{11}$ 
The authors thank the British Paediatric Surveillance Unit for providing the mechanism for data capture and the paediatricians and laboratory investigators for reporting cases and completing questionnaires. This work was supported by the (Children's Hospital Research in Sheffield) Fund.

1 Roe CR, Coates PM. Mitochondrial fatty acid oxidation disorders. In: Scriver CR, Beaudet AL, Sly WS, Valle D, eds. The metabolic and molecular bases of inherited disease. New York: McGraw Hill, 1995:1501-34.

2 Tanaka K, Gregersen N, Ribes A, et al. A survey of the newborn populations in Belgium, Germany, Poland, Czech Republic, Hungary, Bulgaria, Spain, Turkey and Japan for the G985 variant allele with haplotype analysis at the medium-chain acyl-CoA dehydrogenase gene locus: clinical and evolutionary considerations. Pediatr Res 1997;41: 201-9.

3 Seddon HR, Green A, Gray RGF, Leonard JV, Pollitt RJ. Regional variations in medium-chain acyl-CoA dehydrogenase-deficiency. Lancet 1995;345:135-6.

4 Iafolla AK, Thompson RJ, Roe CR. Medium-chain acyl-coenzyme-A dehydrogenase-deficiency-clinical acyl-coenzyme-A dehydrogenase-deficiency-clinica

5 Touma EH, Charpentier C. Medium chain acyl-CoA Touma EH, Charpentier C. Medium chain acyl-CoA

6 Heptinstall LE, Till J, Wraith JE, Besley GTN. Common MCAD mutation in a healthy parent of two affected siblings. F Inherit Metab Dis 1995;18:638-9.

7 Ziadeh R, Hoffman EP, Finegold DN, et al. Medium chain acyl-CoA dehydrogenase deficiency in Pennsylvania: neonatal screening shows high incidence and unexpected mutation frequencies. Pediatr Res 1995;37:675-8.

8 Bartlett K, Eaton SJ, Pourfarzam M. New developments in neonatal screening. Arch Dis Child 1997;77:F151-4.

9 Wilson JMG, Jungner G. Principles of screening for disease. Geneva: World Health Organisation, 1968:26-39.
10 Hall DM, Michel JM. Screening in infancy. Arch Dis Child 1995;72:93-6.

11 Pollitt RJ, Green A, McCabe CJ, et al. Neonatal screening for inborn errors of metabolism: cost, yield and outcome. Health Technol Assess 1997;1:1-202.

12 Surveillance Unit. 10th annual report. London: College of Paediatrics and Child Health, 1996

13 Curtis D, Blakemore AI, Engel PC, et al. Heterogeneity for mutations in medium chain acyl-CoA dehydrogenase deficiency in the UK population. Clin Genet 1991;40:283-6.

14 Wilcken B, Hammond J, Silink M. Morbidity and mortality in medium chain acyl coenzyme A dehydrogenase deficiency. Arch Dis Child 1994;70:410-12.

15 Cone B, Zuffery R, Belin D. The A985G mutation in the medium-chain acyl-CoA dehydrogenase gene: high prevalence in the Swiss populatio
Metab Dis 1995;18:577-85.

16 Fromenty B, Mansouri A Bonnefont JP, et al. Most cases of medium-chain acyl-3 CoA dehydrogenase deficiency escape detection in France. Hum Genet 1996;97:367-8.

17 Gray RGF, Preece MA, Seddon H, Alger S, Elias E, Green A. Two adults with medium-chain acyl-CoA dehydrogenase deficiency - an under diagnosed disorder [abstract]. Society for the study of inborn errors of metabolism, 33rd annual symposium, Toledo, September 1995. Lancaster: Kluwer Academic Publishers, 1995: P115.

18 Dundar M, Lanyon WG, Connor JM. Scottish frequency of the common G985 mutation in the medium-chain the common G985 mutation in the medium-chain acyl-CoA dehydrogenase (MCAD) gene and the role of 13 MCAD deficiency in sudden infant deat
(SIDS). F Inherit Metab Dis 1993;16:991-3.

19 Wilcken B, Carpenter K-H, Hammond J. Neonatal symptoms in medium-chain acylCoA dehydrogenase deficiency. Arch Dis Child 1993;69:292-4.

20 Kirk JM, Laing IA, Smith N, Uttley WS. Neonatal presentation of medium-chain acyl-CoA dehydrogenase deficiency in two families. $\mathcal{F}$ Inherit Metab Dis 1996;19:370-1 . 